Journal of Applied Mathematics and Stochastic Analysis, 16:1 (2003), 1-17.

Printed in the USA (C)2003 by North Atlantic Science Publishing Company

\title{
BSDE ASSOCIATED WITH LÉVY PROCESSES AND APPLICATION TO PDIE
}

\author{
K. BAHLALI \\ UFR Sciences \\ UVT, BP 132, 83957 La Garde Cedex, France ${ }^{1}$ \\ CPT, CNRS, Luminy. Case 907 \\ 13288 Marseille Cedex 9, France \\ E-mail: bahlali@univ-tln.fr \\ M. EDDAHBI \\ Université Cadi Ayyad \\ Faculté des Sciences et Techniques ${ }^{2}$ \\ Départment de Math 8 Info., BP 549 \\ Marrakech, Maroc \\ E-mail: eddahbi@fstg-marrakech.ac.ma \\ E. ESSAKY \\ Université Cadi Ayyad \\ Faculté des Sciences Semlalia ${ }^{3}$ \\ Département de Mathématiques, BP 2390 \\ Marrakech, Maroc \\ E-mail: essaky@ucam.ac.ma
}

(Received April, 2002; Revised November, 2002)

We deal with backward stochastic differential equations (BSDE for short) driven by Teugel's martingales and an independent Brownian motion. We study the existence, uniqueness and comparison of solutions for these equations under a Lipschitz as well as a locally Lipschitz conditions on the coefficient. In the locally Lipschitz case, we prove that if the Lipschitz constant $L_{N}$ behaves as $\sqrt{\log (N)}$ in the ball $B(0, N)$, then the corresponding BSDE has a unique solution which depends continuously on the on the coefficient and the terminal data. This is done with an unbounded terminal data. As application, we give a probabilistic interpretation for a large class of partial differential integral equations (PDIE for short).

Keywords. Backward Stochastic Differential Equations, Lévy Processes, Teugel's Martingales, Partial Differential Integral Equations, Clark-Ocone Formula.

AMS (MOS) subject classification: 60H10, 60H15

\footnotetext{
${ }^{1}$ Supported by CMEP, 077/2001.

${ }^{2}$ Supported by CNRST Maroc/CNRS France, 8310-2000 and TWAS grant 98-199 RG/MATHS/AF/AC.

${ }^{3}$ Supported by CMIFM, A.I. ${ }^{\circ}$ MA/01/02.
} 


\section{Introduction}

Since the paper [8] of Pardoux and Peng, several works have been devoted to the study of BSDEs as well as to their applications. This is due to the connections of BSDEs with stochastic optimal control and stochastic games (Hamadène and Lepeltier [3]) as well as to mathematical finance (El Karoui et al. [4]). Backward stochastic differential equations also appear as a powerful tool in partial differential equations where they provide probabilistic formulas for their solutions (Peng [10], Pardoux and Peng [9]). A solution of a classical BSDE is a pair of adapted processes $(Y, Z)$ satisfying:

$$
Y_{t}=\xi+\int_{t}^{T} f\left(s, Y_{s}, Z_{s}\right) d s-\int_{t}^{T} Z_{s} d W_{s}
$$

When the coefficient $f$ is uniformly Lipschitz, the BSDE (1.1) has a unique solution. The proof is mainly based on the Itô martingale representation theorem.

In Nualart and Schoutens [6], a martingale representation theorem associated to Lévy processes was proved. It then is natural to extend equations (1.1) to BSDE's driven by a Lévy process (Nualart and Schoutens [7]). In their paper [7], the authors proved the existence and uniqueness of solutions, under Lipschitz conditions on the coefficient.

In this paper, we deal with BSDE driven by both a standard Brownian motion and an independent Lévy process and having a Lipschitz, or more generally, a locally Lipschitz coefficient. In the locally Lipschitz case, we prove that if the Lipschitz constant $L_{N}$ behaves as $\sqrt{\log (N)}$ in the ball $B(0, N)$, then the corresponding BSDE has a unique solution. We don't impose any boundedness condition on the terminal data. It will be assumed square integrable only. Moreover, a comparison theorem as well as a stability of solutions are established in this setting. Our results extend in particular those of ([1], [2]) to BSDE driven by a Lévy process. As an application, we give a probabilistic interpretation for a large class of partial differential integral equations.

The paper is organized as follows. In Section 2, we introduce some notations and assumptions. Section 3 is devoted to the proof of existence, uniqueness and comparison results for BSDE driven by a Lévy process, under Lipschitz conditions. Those equations are also discussed under locally Lipschitz conditions in Section 4. In Section 5, we include an application to PDIE.

\section{Preliminaries and Notations}

Let $\left(\Omega, \mathcal{F}, \mathbb{P}, \mathcal{F}_{t}, W_{t}, L_{t}: t \in[0, T]\right)$ be a complete Wiener-Lévy space in $\mathbb{R} \times \mathbb{R} \backslash\{0\}$, with Lévy measure $\nu$, i.e. $(\Omega, \mathcal{F}, \mathbb{P})$ is a complete probability space, $\left\{\mathcal{F}_{t}: t \in[0, T]\right\}$ is a right-continuous increasing family of complete sub $\sigma$-algebras of $\mathcal{F},\left\{W_{t}: t \in[0, T]\right\}$ is a standard Wiener process in $\mathbb{R}$ with respect to $\left\{\mathcal{F}_{t}: t \in[0, T]\right\}$ and $\left\{L_{t}: t \in[0, T]\right\}$ is a $\mathbb{R}$-valued Lévy process of the form $L_{t}=b t+\ell_{t}$ independent of $\left\{W_{t}: t \in[0, T]\right\}$, corresponding to a standard Lévy measure $\nu$ satisfying the following conditions :

i) $\int_{\mathbb{R}}\left(1 \wedge y^{2}\right) \nu(d y)<\infty$,

ii) $\int_{]_{-\varepsilon, \varepsilon\left[c^{c}\right.}} e^{\lambda|y|} \nu(d y)<\infty$, for every $\varepsilon>0$ and for some $\lambda>0$.

We assume that

$$
\mathcal{F}_{t}=\sigma\left(L_{s}, s \leq t\right) \vee \sigma\left(W_{s}, s \leq t\right) \vee \mathcal{N}
$$


where $\mathcal{N}$ denotes the totality of $\mathbb{P}$-null sets and $\mathcal{G}_{1} \vee \mathcal{G}_{2}$ denotes the $\sigma$-field generated by $\mathcal{G}_{1} \cup \mathcal{G}_{2}$.

Let $\mathcal{H}^{2}$ denote the space of real valued, square integrable and $\mathcal{F}_{t}$-progressively measurable processes $\phi=\left\{\phi_{t}: t \in[0, T]\right\}$ such that

$$
\|\phi\|^{2}=\mathbb{E} \int_{0}^{T}\left|\phi_{t}\right|^{2} d t<\infty .
$$

and denote by $\mathcal{P}^{2}$ the subspace of $\mathcal{H}^{2}$ formed by the predictable processes.

Let $l^{2}$ be the space of real valued sequences $\left(x_{n}\right)_{n \geq 0}$ such that $\sum_{i=0}^{\infty} x_{i}^{2}$ is finite. We shall denote by $\mathcal{H}^{2}\left(l^{2}\right)$ and $\mathcal{P}^{2}\left(l^{2}\right)$ the corresponding spaces of $l^{2}$-valued processes equipped with the norm

$$
\|\phi\|^{2}=\sum_{i=0}^{\infty} \mathbb{E} \int_{0}^{T}\left|\phi_{t}^{(i)}\right|^{2} d t
$$

Let us define:

(A.1) a terminal value $\xi \in L^{2}\left(\Omega, \mathcal{F}_{T}, \mathbb{P}\right)$.

(A.2) a process $f$, which is a map $f:[0, T] \times \Omega \times \mathbb{R} \times \mathbb{R} \times l^{2} \longrightarrow \mathbb{R}$, such that

(i) $f$ is progressively measurable also $f(., 0,0,0) \in \mathcal{H}^{2}$.

(ii) There exists $L>0$ such that

$$
\left|f(t, \omega, y, u, z)-f\left(t, \omega, y^{\prime}, u^{\prime}, z^{\prime}\right)\right| \leq L\left(\left|y-y^{\prime}\right|+\left|u-u^{\prime}\right|+\left\|z-z^{\prime}\right\|\right) .
$$

We recall the Itô formula for càdlàg semimartingales.

\subsection{Itô's formula}

Let $X=\left\{X_{t}: t \in[0, T]\right\}$ be a càdlàg semimartingale, with quadratic variation denoted by $[X]=\left\{[X]_{t}: t \in[0, T]\right\}$ and let $F$ be a $\mathcal{C}^{2}$ real valued function. Then $F(X)$ is also a semimartingale and the following formula holds:

$$
\begin{aligned}
F\left(X_{t}\right)= & F\left(X_{0}\right)+\int_{0}^{t} F^{\prime}\left(X_{s-}\right) d X_{s}+\frac{1}{2} \int_{0}^{T} F^{\prime \prime}\left(X_{s}\right) d[X]_{s}^{c} \\
& +\sum_{0<s \leq t}\left\{F\left(X_{s}\right)-F\left(X_{s-}\right)-F^{\prime}\left(X_{s-}\right) \Delta X_{s}\right\} .
\end{aligned}
$$

where $[X]^{c}$ (sometimes denoted by $\langle X\rangle$ ) is the continuous part of the quadratic variation $[X]$. We also note that in the case where $F(x)=x^{2}$, the formula (2.1) takes the form

$$
X_{t}^{2}=X_{0}^{2}+\int_{0}^{t} 2 X_{s-} d X_{s}+\int_{0}^{t} d[X]_{s}
$$

Moreover if $X$ and $Y$ are two càdlàg semimartingales then we have

$$
X_{t} Y_{t}=X_{0} Y_{0}+\int_{0}^{t} X_{s-} d Y_{s}+\int_{0}^{t} Y_{s-} d X_{s}+\int_{0}^{t} d[X, Y]_{s} .
$$

where $[X, Y]$ stands for the quadratic covariation of $X, Y$ also called the bracket process. For a complete survey in this topic we refer to Protter [11]. 


\subsection{Predictable representation}

We denote by $\left(H^{(i)}\right)_{i \geq 1}$ the Teugel's Martingales associated with the Lévy process $\left\{L_{t}: t \in[0, T]\right\}$. More precisely

$$
H_{t}^{(i)}=c_{i, i} Y_{t}^{(i)}+c_{i, i-1} Y_{t}^{(i-1)}+\ldots+c_{i, 1} Y_{t}^{(1)},
$$

where $Y_{t}^{(i)}=L_{t}^{(i)}-\mathbb{E}\left[L_{t}^{(i)}\right]=L_{t}^{(i)}-t \mathbb{E}\left[L_{1}^{(i)}\right]$ for all $i \geq 1$ and $L_{t}^{(i)}$ are power-jump processes. That is, $L_{t}^{(1)}=L_{t}$ and $L_{t}^{(i)}=\sum_{0<s \leq t}\left(\Delta L_{t}\right)^{i}$ for $i \geq 2$. It was shown in Nualart and Schoutens [6] that the coefficients $c_{i, k}$ correspond to the orthonormalization of the polynomials $1, x, x^{2}, \ldots$ with respect to the measure $\mu(d x)=x^{2} \nu(d x)+\sigma^{2} \delta_{0}(d x)$ :

$$
q_{i-1}=c_{i, i} x^{i-1}+c_{i, i-1} x^{i-2}+\ldots+c_{i, 1} .
$$

We set

$$
p_{i}(x)=x q_{i-1}(x)=c_{i, i} x^{i}+c_{i, i-1} x^{i-1}+\ldots+c_{i, 1} x .
$$

The martingales $\left(H^{(i)}\right)_{i \geq 1}$ can be chosen to be pairwise strongly orthonormal martingales. More details, in this subject, can be found in Nualart and Schoutens [6].

The main tool in the theory of BSDEs is the martingale representation theorem, which is well known for martingales which are adapted to the filtration of the Brownian motion or that of Poisson point process (e.g Situ [13]) or that of a Poisson random measure (e.g Ouknine [12]). A more general and interesting martingale representation theorem (proven by different ways) appeared recently in Løkka [5] and in Nualart and Schoutens [7].

Proposition 2.1: Let $\left\{M_{t}: t \in[0, T]\right\}$ be a square integrable martingale which is adapted to the filtration $\mathcal{F}_{t}$ defined above. Then, there exist $U \in \mathcal{P}^{2}$ and $Z \in \mathcal{P}^{2}\left(l^{2}\right)$ such that

$$
M_{t}=\mathbb{E}\left[M_{t}\right]+\int_{0}^{t} U_{s} d W_{s}+\sum_{i=1}^{\infty} \int_{0}^{t} Z_{s}^{(i)} d H_{s}^{(i)}
$$

Proof. The Proof follows by combining the result of Løkka [5] (Theorem 5) and that of Nualart and Schoutens [6].

We denote by $\mathcal{E}$ the set of $\mathbb{R} \times \mathbb{R} \times l^{2}$-valued processes $(Y, U, Z)$ defined on $\mathbb{R}_{+} \times \Omega$ which are $\mathcal{F}_{t}$-adapted and such that:

$$
\|(Y, U, Z)\|^{2}=\mathbb{E}\left(\sup _{0 \leq t \leq T}\left|Y_{t}\right|^{2}+\int_{0}^{T}\left|U_{s}\right|^{2} d s+\int_{0}^{T}\left\|Z_{s}\right\|^{2} d s\right)<+\infty .
$$

The couple $(\mathcal{E},\|\cdot\|)$ is then a Banach space.

We now introduce our BSDE. Given a data $(f, \xi)$ we want to solve the following stochastic integral equation, which we denote by Equation $(f, \xi)$ :

$$
Y_{t}=\xi+\int_{t}^{T} f\left(s, Y_{s-}, U_{s}, Z_{s}\right) d s-\int_{t}^{T} U_{s} d W_{s}-\sum_{i=1}^{\infty} \int_{t}^{T} Z_{s}^{(i)} d H_{s}^{(i)}
$$

Definition 2.2: A solution of equation $E q(f, \xi)$ is a triple $(Y, U, Z)$ which belongs to the space $(\mathcal{E},\|\|$.$) and satisfies E q(f, \xi)$. 


\section{BSDE Driven by Lévy Processes}

\subsection{Existence and uniqueness of solutions}

Theorem 3.1: Let the assumptions (A.1), (A.2) hold. Assume moreover that $\xi$ is a square integrable random variable which is $\mathcal{F}_{T}$-measurable. Then $E q(f, \xi)$ has a unique solution.

Proof: Uniqueness. Let $(Y, U, Z)$ and $(\widetilde{Y}, \widetilde{U}, \widetilde{Z})$ be two solutions of equation $E q(f, \xi)$. By Itô's formula 2.2, we have

$$
\begin{aligned}
& \mathbb{E}\left|Y_{t}-\widetilde{Y}_{t}\right|^{2}+\mathbb{E} \int_{t}^{T}\left|U_{s}-\widetilde{U}_{s}\right|^{2} d s+\mathbb{E} \int_{t}^{T}\left\|Z_{s}-\widetilde{Z}_{s}\right\|^{2} d s \\
= & 2 \mathbb{E} \int_{t}^{T}\left(Y_{s-}-\widetilde{Y}_{s-}\right)\left[f\left(s, Y_{s-}, U_{s}, Z_{s}\right)-f\left(s, \widetilde{Y}_{s-}, \widetilde{U}_{s}, \widetilde{Z}_{s}\right)\right] d s,
\end{aligned}
$$

Since $f$ is $L$-Lipschitz, we get

$$
\begin{aligned}
\mathbb{E}\left|Y_{t}-\widetilde{Y}_{t}\right|^{2} & +\left(1-\frac{2 L}{\beta^{2}}\right) \mathbb{E} \int_{t}^{T}\left|U_{s}-\widetilde{U}_{s}\right|^{2} d s+\left(1-\frac{2 L}{\beta^{2}}\right) \mathbb{E} \int_{t}^{T}\left\|Z_{s}-\widetilde{Z}_{s}\right\|^{2} d s \\
& \leq L\left(\beta^{2}+2\right) \mathbb{E} \int_{t}^{T}\left|Y_{s-}-\widetilde{Y}_{s-}\right|^{2} d s
\end{aligned}
$$

where we have used the inequality $2 x y \leq \beta^{2} x^{2}+\frac{y^{2}}{\beta^{2}}$. If we choose $\frac{2 L}{\beta^{2}}=\frac{1}{2}$, we obtain

$$
\mathbb{E}\left|Y_{t}-\widetilde{Y}_{t}\right|^{2}+\mathbb{E} \int_{t}^{T}\left|U_{s}-\widetilde{U}_{s}\right|^{2} d s+\mathbb{E} \int_{t}^{T}\left\|Z_{s}-\widetilde{Z}_{s}\right\|^{2} d s \leq C \mathbb{E} \int_{t}^{T}\left|Y_{s}-\widetilde{Y}_{s}\right|^{2} d s .
$$

Uniqueness now follows from Gronwall's lemma.

Existence. Using the martingale representation theorem (Proposition 2.1), one can prove that the following BSDE

$$
Y_{t}=\xi+\int_{t}^{T} f(s, 0,0,0) d s-\int_{t}^{T} U_{s} d W_{s}-\int_{t}^{T}\left\langle Z_{s}, d H_{s}\right\rangle
$$

has a solution.

Now, define $\left(Y^{n}, U^{n}, Z^{n}\right)$ as follows: $Y^{0}=Z^{0}=U^{0}=0$ and $\left(Y^{n+1}, U^{n+1}, Z^{n+1}\right)$ is the unique solution to the BSDE

$$
Y_{t}^{n+1}=\xi+\int_{t}^{T} f\left(s, Y_{s-}^{n}, U_{s}^{n}, Z_{s}^{n}\right) d s-\int_{t}^{T} U_{s}^{n+1} d W_{s}-\int_{t}^{T}\left\langle Z_{s}^{n+1}, d H_{s}\right\rangle,
$$

We shall prove that $\left(Y^{n}, U^{n}, Z^{n}\right)$ is a Cauchy sequence in the Banach space $\mathcal{E}$. To simplify the notations, put :

$$
\bar{Y}_{s}^{n, m}:=Y_{s}^{n}-Y_{s}^{m}, \bar{U}_{s}^{n, m}:=U_{s}^{n}-U_{s}^{m} \text { and } \bar{Z}_{s}^{n, m}:=Z_{s}^{n}-Z_{s}^{m}
$$

and

$$
\bar{f}_{s}^{n, m}:=f\left(s, Y_{s-}^{n}, U_{s}^{n}, Z_{s}^{n}\right)-f\left(s, Y_{s-}^{m}, U_{s}^{m}, Z_{s}^{m}\right) .
$$


Itô's formula (2.2), shows that for every $n<m$

$$
\begin{aligned}
e^{\alpha t}\left|\bar{Y}_{t}^{n+1, m+1}\right|^{2} & +\int_{t}^{T} e^{\alpha s}\left|\bar{U}_{s}^{n+1, m+1}\right|^{2} d s \\
& +\int_{t}^{T} e^{\alpha s}\left\|\bar{Z}_{s}^{n+1, m+1}\right\|^{2} d s+\alpha \int_{t}^{T} e^{\alpha s}\left|\bar{Y}_{s-}^{n+1, m+1}\right|^{2} d s \\
& =2 \int_{t}^{T} e^{\alpha s} \bar{Y}_{s-}^{n+1, m+1} \bar{f}_{s}^{n, m} d s-2 \int_{t}^{T} e^{\alpha s} \bar{Y}_{s-}^{n+1, m+1} \bar{U}_{s}^{n, m} d W_{s} \\
& -2 \int_{t}^{T} e^{\alpha s} \bar{Y}_{s-}^{n+1, m+1}\left\langle\bar{Z}_{s}^{n, m}, d H_{s}\right\rangle-\left(N_{T}-N_{t}\right),
\end{aligned}
$$

where $\left\{N_{t}: t \in[0, T]\right\}$ is a martingale given by

$$
N_{t}=\sum_{i=1}^{\infty} \sum_{j=1}^{\infty} \int_{0}^{t} e^{\alpha s} \bar{Z}_{s}^{n+1, m+1,(i)} \bar{Z}_{s}^{n+1, m+1,(j)}\left(d\left[H^{(i)}, H^{(j)}\right]_{s}-d\left\langle H^{(i)}, H^{(j)}\right\rangle_{s}\right) .
$$

Taking the expectation and using the fact that $\left\langle H^{(i)}, H^{(j)}\right\rangle=\delta_{i, j} t$, we get

$$
\begin{aligned}
\mathbb{E} e^{\alpha t}\left|\bar{Y}_{t}^{n+1, m+1}\right|^{2} & +\mathbb{E} \int_{t}^{T} e^{\alpha s}\left|\bar{U}_{s}^{n+1, m+1}\right|^{2} d s \\
& +\mathbb{E} \int_{t}^{T} e^{\alpha s}\left\|\bar{Z}_{s}^{n+1, m+1}\right\|^{2} d s+\alpha \mathbb{E} \int_{t}^{T} e^{\alpha s}\left|\bar{Y}_{s-}^{n+1, m+1}\right|^{2} d s \\
& =2 \mathbb{E} \int_{t}^{T} e^{\alpha s} \bar{Y}_{s-}^{n+1, m+1} \bar{f}_{s}^{n, m} d s
\end{aligned}
$$

Since $f$ is $L$-Lipschitz, we get

$$
\begin{aligned}
& e^{\alpha t} \mathbb{E}\left|\bar{Y}_{t}^{n+1, m+1}\right|^{2}+\int_{t}^{T} e^{\alpha s} \mathbb{E}\left|\bar{U}_{s}^{n+1, m+1}\right|^{2} d s \\
& +\int_{t}^{T} e^{\alpha s} \mathbb{E}\left\|\bar{Z}_{s}^{n+1, m+1}\right\|^{2} d s+\alpha \int_{t}^{T} e^{\alpha s} \mathbb{E}\left|\bar{Y}_{s-}^{n+1, m+1}\right|^{2} d s \\
& \leq 2 L \mathbb{E} \int_{t}^{T} e^{\alpha s}\left|\bar{Y}_{s-}^{n+1, m+1}\right|\left[\left|\bar{Y}_{s-}^{n, m}\right|+\left|\bar{U}_{s}^{n, m}\right|+\left\|\bar{Z}_{s}^{n, m}\right\|\right] d s,
\end{aligned}
$$

and then

$$
\begin{aligned}
e^{\alpha t} \mathbb{E}\left|\bar{Y}_{t}^{n+1, m+1}\right|^{2} & +\int_{t}^{T} e^{\alpha s} \mathbb{E}\left|\bar{U}_{s}^{n+1, m+1}\right|^{2} d s+\int_{t}^{T} e^{\alpha s} \mathbb{E}\left\|\bar{Z}_{s}^{n+1, m+1}\right\|^{2} d s \\
& +\left(\alpha-L^{2} \beta^{2}\right) \int_{t}^{T} e^{\alpha s} \mathbb{E}\left|\bar{Y}_{s-}^{n+1, m+1}\right|^{2} d s \\
& \leq \frac{3}{\beta^{2}} \mathbb{E} \int_{t}^{T} e^{\alpha s}\left(\left|\bar{Y}_{s-}^{n, m}\right|^{2}+\left|\bar{U}_{s}^{n, m}\right|^{2}+\left\|\bar{Z}_{s}^{n, m}\right\|^{2}\right) d s
\end{aligned}
$$


Choosing $\beta$ and $\alpha$ such that $\frac{3}{\beta^{2}}=\frac{1}{2}$ and $\alpha-6 L^{2}=1$, we get

$$
\begin{aligned}
e^{\alpha t} \mathbb{E}\left|\bar{Y}_{t}^{n+1, m+1}\right|^{2} & +\int_{t}^{T} e^{\alpha s} \mathbb{E}\left|\bar{U}_{s}^{n+1, m+1}\right|^{2} d s+\int_{t}^{T} e^{\alpha s} \mathbb{E}\left\|\bar{Z}_{s}^{n+1, m+1}\right\|^{2} d s \\
& \leq \frac{1}{2} \mathbb{E} \int_{t}^{T} e^{\alpha s}\left(\left|\bar{Y}_{s-}^{n, m}\right|^{2}+\left|\bar{U}_{s}^{n, m}\right|^{2}+\left\|\bar{Z}_{s}^{n, m}\right\|^{2}\right) d s
\end{aligned}
$$

It follows immediately, for all $m>n$, that

$$
\mathbb{E} \int_{0}^{T} e^{\alpha s}\left|\bar{Y}_{s-}^{n, m}\right|^{2} d s+\mathbb{E} \int_{0}^{T} e^{\alpha s}\left|\bar{U}_{s}^{n, m}\right|^{2} d s+\mathbb{E} \int_{0}^{T} e^{\alpha s}\left\|\bar{Z}_{s}^{n, m}\right\|^{2} d s \leq \frac{C}{2^{n}} .
$$

Using again Itô's formula and Doob's inequality, it follows that there exists a universal constant $C$ such that

$$
\mathbb{E} \sup _{0 \leq s \leq T}\left|\bar{Y}_{s}^{n, m}\right|^{2}+\mathbb{E} \int_{0}^{T} e^{\alpha s}\left|\bar{U}_{s}^{n, m}\right|^{2} d s+\mathbb{E} \int_{0}^{T} e^{\alpha s}\left\|\bar{Z}_{s}^{n, m}\right\|^{2} d s \leq \frac{C}{2^{n}} .
$$

Consequently, $\left(Y^{n}, U^{n}, Z^{n}\right)$ is a Cauchy sequence in the Banach space $\mathcal{E}$. It is not difficult to show that

$$
(Y, U, Z)=\lim _{n \rightarrow \infty}\left(Y^{n}, U^{n}, Z^{n}\right)
$$

solves our BSDE.

The following theorem gives a bound for the difference between two solutions of $E q(f, \xi)$. It can be proved by using Itô's formula, the Lipschitz property of $f$ and Gronwall's lemma.

Theorem 3.2: Given standard data $(f, \xi)$ and $(\widetilde{f}, \widetilde{\xi})$, let $(Y, U, Z)$ and $(\widetilde{Y}, \widetilde{U}, \widetilde{Z})$, be the unique solution the equation $E q(f, \xi)$ and $E q(\widetilde{f}, \widetilde{\xi})$ respectively. Then

$$
\begin{array}{r}
\mathbb{E} \int_{0}^{T}\left(\left|\widetilde{Y}_{s-}-Y_{s-}\right|^{2}+\left|\widetilde{U}_{s}-U_{s}\right|^{2}+\left\|\widetilde{Z}_{s}-Z_{s}\right\|^{2}\right) d s \\
\leq C\left(\mathbb{E}|\widetilde{\xi}-\xi|^{2}+\mathbb{E} \int_{0}^{T}\left|\widetilde{f}\left(s, Y_{s-}, U_{s}, Z_{s}\right)-f\left(s, Y_{s-}, U_{s}, Z_{s}\right)\right|^{2} d s\right) .
\end{array}
$$

\subsection{Comparison theorem}

In this subsection, we prove a comparison theorem for BSDE driven by Lévy process. This is an important tool in the probabilistic interpretation of viscosity solutions of partial differential equations.

Theorem 3.3: Given standard data $\left(f_{1}, \xi_{1}\right)$ and $\left(f_{2}, \xi_{2}\right)$, suppose that $\xi_{1} \leq \xi_{2}$ and $f_{1}(t, y, u, z) \leq f_{2}(t, y, u, z)$ for all $(y, u, z) \in \mathbb{R} \times \mathbb{R} \times l^{2}, d \mathbb{P} \times d t-a . s$. Then $Y_{t}^{f_{1}} \leq Y_{t}^{f_{2}}$, $t \in[0, T]$.

Proof: Set

$$
\bar{Y}_{s}:=Y_{s}^{f_{2}}-Y_{s}^{f_{1}}, \bar{U}_{s}:=U_{s}^{f_{2}}-U_{s}^{f_{1}}, \bar{Z}_{s}:=Z_{s}^{f_{2}}-Z_{s}^{f_{1}}, \bar{\xi}:=\xi_{2}-\xi_{1},
$$


and

$$
\bar{f}_{s}:=f_{2}\left(s, Y_{s-}^{f_{2}}, U_{s}^{f_{2}}, Z_{s}^{f_{2}}\right)-f_{1}\left(s, Y_{s-}^{f_{2}}, U_{s}^{f_{2}}, Z_{s}^{f_{2}}\right) .
$$

We define three stochastic processes as follows

$$
\begin{aligned}
\alpha_{s} & = \begin{cases}\bar{Y}_{s-}^{-1}\left(f_{1}\left(s, Y_{s-}^{f_{2}}, U_{s}^{f_{2}}, Z_{s}^{f_{2}}\right)-f_{1}\left(s, Y_{s-}^{f_{1}}, U_{s}^{f_{2}}, Z_{s}^{f_{2}}\right)\right) & \text { if } \bar{Y}_{s-} \neq 0 \\
0 & \text { if } \bar{Y}_{s-}=0,\end{cases} \\
\beta_{s} & = \begin{cases}\bar{U}_{s}^{-1}\left(f_{1}\left(s, Y_{s-}^{f_{1}}, U_{s}^{f_{2}}, Z_{s}^{f_{2}}\right)-f_{1}\left(s, Y_{s-}^{f_{1}}, U_{s}^{f_{1}}, Z_{s}^{f_{2}}\right)\right) & \text { if } \bar{U}_{s} \neq 0 \\
0 & \text { if } \bar{U}_{s}=0,\end{cases}
\end{aligned}
$$

and for all $i \in \mathbb{N}^{*}$ let $\widetilde{Z}^{(i)}$ denote the $l^{2}$-valued stochastic process such that its $i$ first components are equal to those of $Z^{f_{2}}$ and its $\mathbb{N}^{*} \backslash\{1,2, \ldots, i\}$ last components are equal to those of $Z^{f_{1}}$. With this notation, we define for $i \in \mathbb{N}^{*}$

$$
\gamma_{s}^{(i)}= \begin{cases}\left(\bar{Z}_{s}^{(i)}\right)^{-1}\left(f_{1}\left(s, Y_{s-}^{f_{1}}, U_{s}^{f_{1}}, \widetilde{Z}_{s}^{(i)}\right)-f_{1}\left(s, Y_{s-}^{f_{1}}, U_{s}^{f_{1}}, \widetilde{Z}_{s}^{(i-1)}\right)\right) & \text { if } \bar{Z}_{s}^{(i)} \neq 0 \\ 0 & \text { if } \bar{Z}_{s}^{(i)}=0 .\end{cases}
$$

It is clear that

$$
\left\langle\gamma_{s}, \bar{Z}_{s}\right\rangle=\left(f_{1}\left(s, Y_{s-}^{f_{1}}, U_{s}^{f_{1}}, Z_{s}^{f_{2}}\right)-f_{1}\left(s, Y_{s-}^{f_{1}}, U_{s}^{f_{1}}, Z_{s}^{f_{1}}\right)\right)
$$

and the processes $\left\{\alpha_{t}: t \in[0, T]\right\},\left\{\beta_{t}: t \in[0, T]\right\}$ and $\left\{\gamma_{t}: t \in[0, T]\right\}$ are progressively measurable and bounded.

For $0 \leq s \leq t \leq T$, let

$$
\begin{gathered}
M_{t}^{H, W}:=\int_{0}^{t} \beta_{s} d W_{s}+\int_{0}^{t}\left\langle\gamma_{s}, d H_{s}\right\rangle \\
\Gamma_{s, t}:=\exp \left[\int_{s}^{t}\left(\alpha_{r} d r-d\left[M_{.}^{H, W}\right]_{r}^{c}+d M_{r}^{H, W}\right)\right] \prod_{s<r \leq t}\left(1+\Delta M_{r}^{H, W}\right) \exp \left(-\Delta M_{r}^{H, W}\right) .
\end{gathered}
$$

Using Itô's formula (2.1) one can see that $\left\{\Gamma_{s, r}: r \in[s, T]\right\}$ satisfies the stochastic linear equation

$$
\Gamma_{s, t}=1+\int_{s}^{t} \Gamma_{s, r-} d M_{r}^{H, W}+\int_{s}^{t} \Gamma_{s, r-} \alpha_{r} d r .
$$

Since

$$
\begin{aligned}
\bar{Y}_{t}= & \bar{\xi}+\int_{t}^{T}\left(\alpha_{r} \bar{Y}_{r-}+\beta_{r} \bar{U}_{r}+\left\langle\gamma_{r}, \bar{Z}_{r}\right\rangle\right) d r \\
& +\int_{t}^{T} \bar{f}_{r} d r-\int_{t}^{T} \bar{U}_{r} d W_{s}-\int_{t}^{T}\left\langle\bar{Z}_{r}, d H_{r}\right\rangle,
\end{aligned}
$$

we use formula (2.3) and relation (3.1) to show that for all $0 \leq s \leq t \leq T$

$$
\begin{aligned}
\bar{Y}_{s}= & \Gamma_{s, t} \bar{Y}_{t}+\int_{s}^{t} \Gamma_{s, r-} \bar{f}_{r} d r \\
& -\int_{s}^{t} \Gamma_{s, r-}\left(\bar{U}_{r}+\beta_{r} \bar{Y}_{r-}\right) d W_{s}-\int_{s}^{t} \Gamma_{s, r-}\left\langle\gamma_{r} \bar{Y}_{r-}+\bar{Z}_{r}, d H_{r}\right\rangle \\
& +\sum_{i=1}^{\infty} \int_{s}^{t} \Gamma_{s, r-} \gamma_{r}^{(i)} \bar{Z}_{r}^{(i)}\left(d\left[H^{(i)}\right]_{r}-d<H^{(i)}>_{r}\right) .
\end{aligned}
$$


Since the last three terms in the right-hand of the above equation are martingales, we deduce that

$$
\bar{Y}_{s}=\mathbb{E}\left(\Gamma_{s, t} \bar{Y}_{t}+\int_{s}^{t} \Gamma_{s, r-} \bar{f}_{r} d r / \mathcal{F}_{s}\right) .
$$

Hence, the result follows, for $t=T$, by the positivity of $\bar{\xi}$ and $\bar{f}$.

\section{BSDE with Locally Lipschitz Coefficient}

The aim of this section is to prove the existence and uniqueness of solutions for BSDE with locally Lipschitz generator. More precisely, we assume that the following conditions hold:

H.1) $f$ is continuous in $(y, u, z)$ for almost all $(t, \omega)$,

H.2) there exists $K>0$ and $0 \leq \alpha<1$ such that $|f(t, \omega, u, y, z)| \leq K\left(1+|y|^{\alpha}+|u|^{\alpha}+\right.$ $\left.\|z\|^{\alpha}\right)$.

H.3) for every $N \in \mathbb{N}$, there exists a constant $L_{N}>0$ such that $\left|f(t, \omega, y, u, z)-f\left(t, \omega, y^{\prime}, u^{\prime}, z^{\prime}\right)\right| \leq L_{N}\left(\left|y-y^{\prime}\right|+\left|u-u^{\prime}\right|+\left\|z-z^{\prime}\right\|\right), \mathbb{P}-$ a.s., a.e. $t \in[0, T]$

and $\forall y, y^{\prime}, u, u^{\prime}, z, z^{\prime}$ such that $|y| \leq N,\left|y^{\prime}\right| \leq N,|u| \leq N,\left|u^{\prime}\right| \leq N,\|z\| \leq N$, $\left\|z^{\prime}\right\| \leq N$.

When the assumptions H.1) and H.2) are satisfied, we can define the family of seminorms $\left(\rho_{n}(f)\right)_{n}$

$$
\rho_{n}(f)=\left(\mathbb{E} \int_{0}^{T} \sup _{|y|,|u|,|| z \| \leq n}|f(s, y, u, z)|^{2} d s\right)^{\frac{1}{2}} .
$$

We denote by Liploc (resp. Lip) the set of processes $f$ satisfying H.1)-H.2) which are locally Lipschitz, i.e. satisfy the assumption H.3), (resp. globally Lipschitz) with respect to $(y, u, z)$.

Lip loc, $\alpha$ denotes the subset of those processes $f$ which belong to Lip loc and which satisfy H.2).

The main results are the following

Theorem 4.1: (Existence and uniqueness). Let $f \in$ Lip $_{l o c, \alpha}$ and $\xi$ be a square integrable random variable. Then equation $E q(f, \xi)$ has a unique solution if $L_{N} \leq$ $L+\sqrt{\log (N)}$, where $L$ is some positive constant.

We give now a stability result for the solution with respect to the data $(f, \xi)$. Roughly speaking, if $f_{n}$ converges to $f$ in the metric defined by the family of semi-norms $\left(\rho_{N}\right)$ and $\xi_{n}$ converges to $\xi$ in $L^{2}(\Omega)$ then $\left(Y^{n}, U^{n}, Z^{n}\right)$ converges to $(Y, U, Z)$ in $\mathcal{E}$. Let $\left(f_{n}\right)$ be a sequence of functions which are $\mathcal{F}_{t}$-progressively measurable for each $n$. Let $\left(\xi_{n}\right)_{n \geq 1}$ be a sequence of random variables which are $\mathcal{F}_{T}$-measurable for each $n$ and such that $\mathbb{E}\left|\xi_{n}\right|^{2}<\infty$. We will assume that for each $n$, the $\operatorname{BSDE} E q\left(f_{n}, \xi_{n}\right)$ corresponding to the data $\left(f_{n}, \xi_{n}\right)$ has a (not necessarily unique) solution. Each solution of the equation $E q\left(f_{n}, \xi_{n}\right)$ will be denoted by $\left(Y^{f_{n}}, Z^{f_{n}}\right)$.

We suppose also that the following assumptions H.4), H.5) and H.6) are fulfilled,

H.4) For every $N, \rho_{N}\left(f_{n}-f\right) \longrightarrow 0$ as $n \rightarrow \infty$. 
H.5) $\mathbb{E}\left|\xi_{n}-\xi\right|^{2} \longrightarrow 0$ as $n \rightarrow \infty$.

H.6) There exist $K>0$ such that,

$$
\sup _{n}\left|f_{n}(t, \omega, y, u, z)\right| \leq K\left(1+|y|^{\alpha}+|u|^{\alpha}+\|z\|^{\alpha}\right) \quad \mathbb{P} \text {-a.s., a.e. } t \in[0, T] .
$$

Theorem 4.2:(Stability). Let $f$ and $\xi$ be as in Theorem 4.1. Assume that $\left(f_{n}, \xi_{n}\right)$ satisfies H.4), H.5) and H.6). Then we have

$$
\lim _{n \rightarrow+\infty}\left(\mathbb{E} \sup _{0 \leq t \leq T}\left|Y_{t}^{f_{n}}-Y_{t}\right|^{2}+\mathbb{E} \int_{0}^{T}\left|U_{s}^{f_{n}}-U_{s}\right|^{2} d s+\mathbb{E} \int_{0}^{T}\left\|Z_{s}^{f_{n}}-Z_{s}\right\|^{2} d s\right)=0 .
$$

To prove Theorems 4.1 and 4.2 we need the two following lemmas.

Lemma 4.3: Let $\xi^{1}$, $\xi^{2}$ be two d-dimensional square integrable random variables which are $\mathcal{F}_{T}$-measurable. Let $f_{1}$ and $f_{2}$ be two functions which satisfy H.1), H.2). Let $\left(Y^{f_{1}}, U^{f_{1}}, Z^{f_{1}}\right)\left[\right.$ resp. $\left.\left(Y^{f_{2}}, U^{f_{2}}, Z^{f_{2}}\right)\right]$ be a solution of the BSDE Eq $\left(f_{1}, \xi^{1}\right)[$ resp. $\left.E q\left(f_{2}, \xi^{2}\right)\right]$. Then for every locally Lipschitz function $f$ and every $N>1$, the following estimates hold

$$
\begin{aligned}
& \mathbb{E} \int_{0}^{T}\left|U_{s}^{f_{1}}-U_{s}^{f_{2}}\right|^{2} d s+\mathbb{E} \int_{0}^{T}\left\|Z_{s}^{f_{1}}-Z_{s}^{f_{2}}\right\|^{2} d s \\
\leq & C\left(K, \xi^{1}, \xi^{2}\right)\left\{\mathbb{E}\left(\left|\xi^{1}-\xi^{2}\right|^{2}\right)+\left[\mathbb{E} \int_{0}^{T}\left|Y_{s}^{f_{1}}-Y_{s}^{f_{2}}\right|^{2} d s\right]^{\frac{1}{2}}\right\}
\end{aligned}
$$

and

$$
\begin{aligned}
\mathbb{E}\left(\left|Y_{s}^{f_{1}}-Y_{s}^{f_{2}}\right|^{2}\right) \leq & C_{1}\left[\mathbb{E}\left(\left|\xi^{1}-\xi^{2}\right|^{2}\right)+\rho_{N}^{2}\left(f_{1}-f\right)+\rho_{N}^{2}\left(f-f_{2}\right)\right. \\
& \left.+\frac{C\left(K, \xi^{1}, \xi^{2}\right)}{\left(2 L_{N}+2 L_{N}^{2}\right) N^{2(1-\alpha)}}\right] \exp \left(\left(2 L_{N}+2 L_{N}^{2}\right)(T-s)\right),
\end{aligned}
$$

where $C\left(K, \xi^{1}, \xi^{2}\right)$ is a constant which depends on $K, \mathbb{E}\left|\xi^{1}\right|^{2}$ and $\mathbb{E}\left|\xi^{2}\right|^{2}$, and $C_{1}$ is a universal constant.

Proof: The first inequality follows from Itô's formula and Schwarz inequality. We shall prove the second one. Let $<,>$ denote the inner product in $\mathbb{R}^{d}$.

We set

$$
\bar{Y}_{s}:=Y_{s}^{f_{1}}-Y_{s}^{f_{2}}, \bar{U}_{s}:=U_{s}^{f_{1}}-U_{s}^{f_{2}} \text { and } \bar{Z}_{s}:=Z_{s}^{f_{1}}-Z_{s}^{f_{2}}
$$

and

$$
\bar{f}_{s}:=f_{1}\left(s, Y_{s-}^{f_{1}}, U_{s}^{f_{1}}, Z_{s}^{f_{1}}\right)-f_{2}\left(s, Y_{s-}^{f_{2}}, U_{s}^{f_{2}}, Z_{s}^{f_{2}}\right) .
$$

By Itô's formula we have

$$
\begin{aligned}
\left|\bar{Y}_{t}\right|^{2} & +\int_{t}^{T}\left|\bar{U}_{s}\right|^{2} d s+\int_{t}^{T}\left\|\bar{Z}_{s}\right\|^{2} d s=\left|\xi^{1}-\xi^{2}\right|^{2}+2 \int_{t}^{T} \bar{Y}_{s-} \bar{f}_{s} d s-2 \int_{t}^{T} \bar{Y}_{s-} \bar{U}_{s} d W_{s} \\
& -2 \sum_{i=1}^{\infty} \int_{t}^{T} \bar{Y}_{s-} \bar{Z}_{s}^{(i)} d H_{s}^{(i)}-\sum_{i=1}^{\infty} \sum_{j=1}^{\infty} \int_{t}^{T} \bar{Z}_{s}^{(i)} \bar{Z}_{s}^{(j)} d\left(\left[H^{(i)}, H^{(j)}\right]_{s}-<H^{(i)}, H^{(j)}>_{s}\right) .
\end{aligned}
$$


Using the fact that $\int_{0}^{t} \bar{Z}_{s}^{(i)} \bar{Z}_{s}^{(j)} d\left(\left[H^{(i)}, H^{(j)}\right]_{s}-<H^{(i)}, H^{(j)}>_{s}\right)$ is a martingale and taking the expectation we get

$$
\mathbb{E}\left|\bar{Y}_{t}\right|^{2}+\mathbb{E} \int_{t}^{T}\left|\bar{U}_{s}\right|^{2} d s+\mathbb{E} \int_{t}^{T}\left\|\bar{Z}_{s}\right\| d s=\mathbb{E}\left|\xi^{1}-\xi^{2}\right|^{2}+2 \mathbb{E} \int_{t}^{T}\left\langle\bar{Y}_{s-}, \bar{f}_{s}\right\rangle d s .
$$

Let $\beta$ and $\gamma$ be strictly positive numbers. For a given $N>1$, let $L_{N}$ be the Lipschitz constant of $f$ in the ball $B(0, N)$,

$$
A^{N}:=\left\{(s, \omega) ; \quad\left|Y_{s-}^{f_{1}}\right|^{2}+\left|U_{s}^{f_{2}}\right|^{2}+\left\|Z_{s}^{f_{1}}\right\|^{2}+\left|U_{s}^{f_{1}}\right|^{2}+\left|Y_{s-}^{f_{2}}\right|^{2}+\left\|Z_{s}^{f_{2}}\right\|^{2} \geq N^{2}\right\},
$$

$A^{N, c}:=\Omega \backslash A^{N}$ and denote by $\mathbb{1}_{A}$ the indicator function of the set $A$. We have

$$
\begin{aligned}
\mathbb{E}\left|\bar{Y}_{t}\right|^{2}+\mathbb{E} \int_{t}^{T}\left|\bar{U}_{s}\right|^{2} d s+\mathbb{E} \int_{t}^{T}\left\|\bar{Z}_{s}\right\|^{2} d s & =\mathbb{E}\left|\xi^{1}-\xi^{2}\right|^{2}+2 \mathbb{E} \int_{t}^{T}\left\langle\bar{Y}_{s-}, \bar{f}_{s}\right\rangle\left(\mathbb{1}_{A^{N}}+\mathbb{1}_{A^{N, c}}\right) d s \\
& :=\mathbb{E}\left|\xi^{1}-\xi^{2}\right|^{2}+I_{1}+I_{2}+I_{3}+I_{4},
\end{aligned}
$$

where

$$
\begin{aligned}
& I_{1}:=2 \mathbb{E} \int_{t}^{T}\left\langle\bar{Y}_{s-}, \bar{f}_{s}\right\rangle \mathbb{1}_{A^{N}} d s \\
& I_{2}:=2 \mathbb{E} \int_{t}^{T}\left\langle\bar{Y}_{s-}, f_{1}\left(s, Y_{s-}^{f_{1}}, U_{s}^{f_{1}}, Z_{s}^{f_{1}}\right)-f\left(s, Y_{s-}^{f_{1}}, U_{s}^{f_{1}}, Z_{s}^{f_{1}}\right)\right\rangle \mathbb{1}_{A^{N, c}} d s \\
& I_{3}:=2 \mathbb{E} \int_{t}^{T}\left\langle\bar{Y}_{s-}, f\left(s, Y_{s-}^{f_{1}}, U_{s}^{f_{1}}, Z_{s}^{f_{1}}\right)-f\left(s, Y_{s-}^{f_{2}}, U_{s}^{f_{2}}, Z_{s}^{f_{2}}\right)\right\rangle \mathbb{1}_{A^{N, c}} d s . \\
& I_{4}:=2 \mathbb{E} \int_{t}^{T}\left\langle\bar{Y}_{s-}, f\left(s, Y_{s-}^{f_{2}}, U_{s}^{f_{2}}, Z_{s}^{f_{2}}\right)-f_{2}\left(s, Y_{s-}^{f_{2}}, U_{s}^{f_{2}}, Z_{s}^{f_{2}}\right)\right\rangle \mathbb{1}_{A^{N, c}} d s .
\end{aligned}
$$

It is not difficult to check that

$$
\begin{aligned}
& I_{2} \leq \mathbb{E} \int_{t}^{T}\left|\bar{Y}_{s-}\right|^{2} \mathbb{1}_{A^{N, c}} d s+\rho_{N}^{2}\left(f_{1}-f\right) \\
& I_{4} \leq \mathbb{E} \int_{t}^{T}\left|\bar{Y}_{s-}\right|^{2} \mathbb{1}_{A^{N, c}} d s+\rho_{N}^{2}\left(f-f_{2}\right) .
\end{aligned}
$$

Since $f$ is $L_{N}$-Lipschitz in the ball $B(0, N)$, we get

$$
I_{3} \leq\left(2 L_{N}+\gamma^{2}\right) \mathbb{E} \int_{t}^{T}\left|\bar{Y}_{s-}\right|^{2} \mathbb{1}_{A^{N, c}} d s+\frac{2 L_{N}^{2}}{\gamma^{2}} \mathbb{E} \int_{t}^{T}\left|\bar{U}_{s}\right|^{2} d s+\frac{2 L_{N}^{2}}{\gamma^{2}} \mathbb{E} \int_{t}^{T}\left\|\bar{Z}_{s}\right\|^{2} d s .
$$

To estimate $I_{1}$, we use Hölder's inequality and the fact that

$$
\mathbb{1}_{A^{N}} \leq \frac{\left|Y_{s-}^{f_{1}}\right|^{2}+\left|U_{s}^{f_{2}}\right|^{2}+\left\|Z_{s}^{f_{1}}\right\|^{2}+\left|U_{s}^{f_{1}}\right|^{2}+\left|Y_{s-}^{f_{2}}\right|^{2}+\left\|Z_{s}^{f_{2}}\right\|^{2}}{N^{2}}
$$

to obtain

$$
I_{1} \leq \beta^{2} \mathbb{E} \int_{t}^{T}\left|\bar{Y}_{s}\right|^{2} \mathbb{1}_{A^{N}} d s+\frac{1}{\beta^{2}} \mathbb{E} \int_{t}^{T}\left|\bar{f}_{s}\right|^{2} \mathbb{1}_{A^{N}} d s
$$




$$
\begin{aligned}
\leq & \beta^{2} \mathbb{E} \int_{t}^{T}\left|\bar{Y}_{s-}\right|^{2} \mathbb{1}_{A^{N}} d s+\frac{8 K^{2}}{\beta^{2}} \mathbb{E} \int_{t}^{T}\left(1+\left|Y_{s-}^{f_{2}}\right|^{2 \alpha}+\left|U_{s}^{f_{2}}\right|^{2 \alpha}+\left\|Z_{s}^{f_{2}}\right\|^{2 \alpha}\right) \mathbb{1}_{A^{N}} d s \\
& +\frac{8 K^{2}}{\beta^{2}} \mathbb{E} \int_{t}^{T}\left(1+\left|Y_{s-}^{f_{1}}\right|^{2 \alpha}+\left|U_{s}^{f_{1}}\right|^{2 \alpha}+\left\|Z_{s}^{f_{1}}\right\|^{2 \alpha}\right) \mathbb{1}_{A^{N}} d s \\
\leq & \beta^{2} \mathbb{E} \int_{t}^{T}\left|\bar{Y}_{s-}\right|^{2} \mathbb{1}_{A^{N}} d s+\frac{C\left(K, \xi^{1}, \xi^{2}\right)}{\beta^{2} N^{2(1-\alpha)}} .
\end{aligned}
$$

If we choose $\beta^{2}=2 L_{N}^{2}+2 L_{N}$ and $\gamma^{2}=2 L_{N}^{2}$ then we use the above estimates we have

$$
\begin{aligned}
\mathbb{E}\left|\bar{Y}_{t}\right|^{2} & +\mathbb{E} \int_{t}^{T}\left|\bar{U}_{s}\right|^{2} d s+\mathbb{E} \int_{t}^{T}\left\|\bar{Z}_{s}\right\|^{2} d s \\
\leq & \mathbb{E}\left|\xi^{1}-\xi^{2}\right|^{2}+\left(2 L_{N}^{2}+2 L_{N}+2\right) \mathbb{E} \int_{t}^{T}\left|\bar{Y}_{s}\right|^{2} d s \\
& +\rho_{N}^{2}\left(f_{1}-f\right)+\rho_{N}^{2}\left(f-f_{2}\right)+\frac{C\left(K, \xi^{1}, \xi^{2}\right)}{\beta^{2} N^{2(1-\alpha)}}
\end{aligned}
$$

Using Gronwall's lemma, we get

$$
\begin{aligned}
\mathbb{E}\left|\bar{Y}_{t}\right|^{2} \leq & {\left[\mathbb{E}\left|\xi^{1}-\xi^{2}\right|^{2}+\frac{C\left(K, \xi^{1}, \xi^{2}\right)}{\left(2 L_{N}^{2}+2 L_{N}\right) N^{2(1-\alpha)}}\right] \exp \left(\left[L_{N}^{2}+2 L_{N}\right](T-t)+2\right) } \\
& +\left[\rho_{N}^{2}\left(f_{1}-f\right)+\rho_{N}^{2}\left(f-f_{2}\right)\right] \exp \left(\left[L_{N}^{2}+2 L_{N}\right](T-t)+2\right) .
\end{aligned}
$$

Lemma 4.3 is proved.

Lemma 4.4: Let $f$ be a function which satisfies H.1), H.2) and H.3). Then there exists a sequence of functions $f_{n}$ such that,

(i) a) For each $n, f_{n} \in \operatorname{Lip}_{\alpha}$.

b) $\sup _{n}\left|f_{n}(t, \omega, y, u, z)\right| \leq|f(t, \omega, y, u, z)| \leq K\left(1+|y|^{\alpha}+|u|^{\alpha}+\|z\|^{\alpha}\right) \mathbb{P}-$ a.s., a.e. $t \in[0, T]$.

(ii) For every $N, \rho_{N}\left(f_{n}-f\right) \longrightarrow 0$ as $n \longrightarrow \infty$.

Proof: Let $\psi_{n}$ be a sequence of smooth functions with support in the ball $B(0, n+1)$ and such that $\psi_{n}=1$ in the ball $B(0, n)$. It is not difficult to see that the sequence $\left(f_{n}\right)$ of truncated functions, defined by $f_{n}=f \psi_{n}$, satisfies all the properties quoted in Lemma 4.4 .

Lemma 4.5: Let $f$ and $\xi$ be as in Theorem 4.1. Let $\left(f_{n}\right)$ be the sequence of functions associated to $f$ by Lemma 4.4 and denote by $\left(Y^{f_{n}}, U^{f_{n}}, Z^{f_{n}}\right)$ the solution of equation $E q\left(f_{n}, \xi\right)$. Then,

(i) a) $\sup _{n} \mathbb{E}\left|Y_{t}^{f_{n}}\right|^{2} \leq\left[\mathbb{E}|\xi|^{2}+K\right] \exp (K)=K_{1}$.

b) $\sup _{n} \mathbb{E}\left(\int_{0}^{T}\left|U_{s}^{f_{n}}\right|^{2} d s+\int_{0}^{T}\left\|Z_{s}^{f_{n}}\right\|^{2} d s\right) \leq\left[\mathbb{E}|\xi|^{2}+K\right][2+(K) \exp (K)]=K_{2}$.

(ii) There exists a process $(Y, U, Z) \in \mathcal{E}$ such that

$$
\lim _{n \rightarrow \infty}\left\|\left(Y^{f_{n}}, U^{f_{n}}, Z^{f_{n}}\right)-(Y, U, Z)\right\|=0
$$


Proof of Lemma 4.5: For simplicity,we assume $L=0$. Assertion (i) follows from standard arguments of BSDE. Let us prove (ii). First, assume that $L_{N} \leq \sqrt{\frac{(1-\alpha)}{2(T-t)} \log (N)}$. Then applying Lemma 4.3 to $\left(Y^{f_{1}}, U^{f_{1}}, Z^{f_{1}}, f_{1}, \xi^{1}\right)=\left(Y^{f_{n}}, U^{f_{n}}, Z^{f_{n}}, f_{n}, \xi\right)$, $\left(Y^{f_{2}}, U^{f_{2}}, Z^{f_{2}}, f_{2}, \xi^{2}\right)=\left(Y^{f_{m}}, U^{f_{m}}, Z^{f_{m}}, f_{m}, \xi\right)$ and next passing to the limits successively on $n, m, N$ one gets Lemma 4.5. Assume now that $L_{N} \leq \sqrt{\log (N)}$. Let $\delta$ be a strictly positive number such that $\delta<\frac{(1-\alpha)}{2}$. Let $\left(\left[t_{i+1}, t_{i}\right]\right)$ be a subdivision of $[0, T]$ such that $\left|t_{i+1}-t_{i}\right| \leq \delta$. Applying Lemma 4.3 in all the subintervals $\left[t_{i+1}, t_{i}\right]$ we get Lemma 4.5.

Proof of Theorems 4.1 and 4.2:. The uniqueness follows from Lemma 4.3 by letting $f_{1}=f_{2}=f$ and $\xi^{1}=\xi^{2}=\xi$ ). We shall prove the existence of solutions. By Lemma 4.5, there exists $(Y, U, Z) \in \mathcal{E}$ such that $\left\|\left(Y^{f_{n}}, U^{f_{n}}, Z^{f_{n}}\right)-(Y, U, Z)\right\| \rightarrow 0$ as $n \rightarrow \infty$. Thus, we immediately have

$$
\begin{aligned}
\lim _{n \rightarrow \infty} \mathbb{E}\left(\sup _{0 \leq s \leq T}\left|Y_{s}^{f_{n}}-Y_{s}\right|^{2}\right) & =\lim _{n \rightarrow \infty} \mathbb{E} \int_{0}^{T}\left|U_{s}^{f_{n}}-U_{s}\right|^{2} d s \\
& =\lim _{n \rightarrow \infty} \mathbb{E} \int_{0}^{T}\left\|Z^{f_{n}}-Z_{s}\right\|^{2} d s=0 .
\end{aligned}
$$

It remains to prove that $\int_{t}^{T} f_{n}\left(s, Y_{s-}^{f_{n}}, U_{s}^{f_{n}}, Z_{s}^{f_{n}}\right) d s$ converges to $\int_{t}^{T} f\left(s, Y_{s-}, U_{s}, Z_{s}\right) d s$ in probability. Let $N>1$ and denote by $L_{N}$ the Lipschitz constant of $f$ in the ball $B(0, N)$. We put $A_{n}^{N}:=\left\{(s, \omega):\left|Y_{s-}^{f_{n}}\right|+\left|U_{s}^{f_{n}}\right|+|| Z_{s}^{f_{n}}\left\|+\left|Y_{s-}\right|+\left|U_{s}\right|+\right\| Z_{s} \| \geq N\right\}$ and $A_{n}^{N, c}:=\Omega \backslash A_{n}^{N}$. Since $f$ is $L_{N}$-locally Lipschitz, we use the triangle inequality and Lemma 4.4 to obtain

$$
\begin{aligned}
& \mathbb{E}\left|\int_{t}^{T} \bar{f}_{s}^{n} d s\right| \leq \mathbb{E} \int_{t}^{T}\left|f_{n}\left(s, Y_{s-}^{f_{n}}, U_{s}^{f_{n}}, Z_{s}^{f_{n}}\right)-f\left(s, Y_{s-}^{f_{n}}, U_{s}^{f_{n}}, Z_{s}^{f_{n}}\right)\right| d s \\
&+\mathbb{E} \int_{0}^{T}\left|f\left(s, Y_{s-}^{f_{n}}, U_{s}^{f_{n}}, Z_{s}^{f_{n}}\right)-f\left(s, Y_{s-}, U_{s}, Z_{s}\right)\right| d s \\
& \leq \mathbb{E} \int_{0}^{T} \sup _{|y|,|u|,\|z\| \leq N}\left|f_{n}(s, y, u, z)-f(s, y, u, z)\right| d s \\
&+2 K \sup _{n} \mathbb{E} \int_{0}^{T}\left(1+\left|Y_{s-}^{f_{n}}\right|^{\alpha}+\left|U_{s}^{f_{n}}\right|^{\alpha}+\left\|Z_{s}^{f_{n}}\right\|^{\alpha}\right) \mathbb{1}_{A_{n}^{N, c}} d s \\
&+L_{N}\left(\mathbb{E} \int_{0}^{T}\left|\bar{Y}_{s-}^{f_{n}}\right| d s+\mathbb{E} \int_{0}^{T}\left|\bar{U}_{s}^{f_{n}}\right| d s+\mathbb{E} \int_{0}^{T}\left\|\bar{Z}_{s}^{f_{n}}\right\| d s\right) \\
&+K \sup _{n} \mathbb{E} \int_{0}^{T}\left(2+\left|Y_{s-}^{f_{n}}\right|^{\alpha}+\left|U_{s}^{f_{n}}\right|^{\alpha}+\left\|Z_{s}^{f_{n}}\right\|^{\alpha}+\left|Y_{s-}\right|^{\alpha}+\left|U_{s}\right|^{\alpha}+\left\|Z_{s}\right\|^{\alpha}\right) \mathbb{1}_{A_{n}^{N}, c} d s .
\end{aligned}
$$

Since $|x|^{\alpha} \leq 1+|x|$ for each $\alpha \in[0,1[$, we successively use Lemma 4.5 (i) -b), Schwarz inequality, Chebychev inequality, Lemma 4.5 (i) and Fatou's lemma to get

$$
\mathbb{E}\left|\int_{t}^{T} \bar{f}_{s}^{n} d s\right| \leq I_{1}(n)+L_{N} I_{2}(n)+\frac{24 K}{N}\left(1+2\left(K_{1}+K_{2}\right)\right),
$$

where $K_{1}, K_{2}$ denote the two constant defined in Lemma 4.5 (ii) and where

$$
I_{1}(n):=\mathbb{E} \int_{0}^{T} \sup _{|y|,|u|,\|z\| \leq N}\left|f_{n}(s, y, u, z)-f(s, y, u, z)\right| d s
$$


and

$$
I_{2}(n):=\mathbb{E} \int_{0}^{T}\left|\bar{Y}_{s-}^{f_{n}}\right| d s+\mathbb{E} \int_{0}^{T}\left|\bar{U}_{s}^{f_{n}}\right| d s+\mathbb{E} \int_{0}^{T}\left\|\bar{Z}_{s}^{f_{n}}\right\| d s .
$$

Lemma 4.5 (ii) shows that $\lim _{n \rightarrow \infty} I_{1}(n)=0$. We shall prove that $\lim _{n \rightarrow \infty} I_{2}(n)=0$. From Lemma 4.5 we have

$$
\lim _{n \rightarrow \infty} \mathbb{E} \int_{0}^{T}\left(\left|\bar{U}_{s}^{n}\right|+\left\|\bar{Z}_{s}^{n}\right\|\right) d s=0
$$

We use Lemma 4.5, Fatou's Lemma and the Lebesgue dominated convergence theorem to show that $\lim _{n \rightarrow \infty} \mathbb{E} \int_{0}^{T}\left|\bar{Y}_{s}^{f_{n}}\right| d s=0$ which shows that equation $E q(f, \xi)$ has at least one solution. Theorem 4.1 is proved. We get Theorem 4.2 by applying Lemma 4.3 to $\left(Y^{f_{1}}, U^{f_{1}}, Z^{f_{1}}, f_{1}, \xi^{1}\right)=(Y, U, Z, f, \xi),\left(Y^{f_{2}}, U^{f_{2}}, Z^{f_{2}}, f_{2}, \xi^{2}\right)=\left(Y^{f_{n}}, U^{f_{n}}, Z^{f_{n}}, f_{n}, \xi\right)$ and by passing to the limits, first on $n$ and next on $N$. The proofs are finished.

\section{Applications to PDIE}

In this section, we give the links between BSDE driven by Lévy process and a family of partial differential integral equation (PDIE). Let $X_{t}=\int_{0}^{t} \sigma\left(X_{s}\right) d W_{s}+L_{t}$, recall that $L_{t}$ is a Lévy process with Lévy measure $\nu$, which takes the form $L_{t}=b t+\ell_{t}$.

We give a technical lemma which will be needed later on.

Lemma 5.1: Let $h: \Omega \times[0, T] \times \mathbb{R} \longrightarrow \mathbb{R}$ be a measurable function such that

$$
|h(s, y)| \leq \theta_{s}\left(y^{2} \wedge|y|\right) \quad \text { a.s. }
$$

where $\left\{\theta_{s}: 0 \leq s \leq T\right\}$ is a nonnegative predictable process such that $\mathbb{E} \int_{0}^{T} \theta_{s} d s<\infty$. Then for each $t \in[0, T]$ we have

$$
\sum_{t<s \leq T} h\left(s, \Delta X_{s}\right)=\sum_{i=1}^{\infty} \int_{t}^{T}\left\langle h(s, .), p_{i}(.)\right\rangle_{L^{2}(\nu)} d H_{s}^{(i)}+\int_{t}^{T} \int_{\mathbb{R}} h(s, y) \nu(d y) d s .
$$

Proof: Since $\Delta X_{t}=\Delta L_{t}$, the proof can be performed as that of Nualart and Schoutens [6].

Now, we apply our result to give a version of Clark-Ocone formula for functions of a Lévy process. Consider the following BSDE

$$
Y_{t}=g\left(X_{T}\right)+\int_{t}^{T} f\left(s, Y_{s-}, U_{s}, Z_{s}\right) d s-\int_{t}^{T} U_{s} d W_{s}-\int_{t}^{T}\left\langle Z_{s}, d H_{s}\right\rangle,
$$

where $\mathbb{E}\left|g\left(X_{T}\right)^{2}\right|<\infty$.

Define

$$
u^{1}(t, x, y)=u(t, x+y)-u(t, x)-\frac{\partial u}{\partial x}(t, x) y,
$$

where $u$ is the solution of the following PDIE

$$
\left\{\begin{array}{l}
\frac{\partial u}{\partial t}(t, x)+\frac{1}{2} \sigma^{2}(x) \frac{\partial^{2} u}{\partial x^{2}}(t, x)+f\left(t, u(t, x), \sigma(x) \frac{\partial u}{\partial x}(t, x),\left(u^{(i)}\right)_{i=1}^{\infty}\right. \\
\quad+\int_{\mathbb{R}} u^{1}(t, x, y) \nu(d y)+\bar{b} \frac{\partial u}{\partial x}(t, x)=0 \\
u(T, x)=g(x),
\end{array}\right.
$$


$\bar{b}=b+\int_{\{|y| \geq 1\}} y \nu(d y)$ and

$$
u^{(1)}(t, x)=\int_{\mathbb{R}} u^{1}(t, x, y) p_{1}(y) \nu(d y)+\frac{\partial u}{\partial x}(t, x)\left(\int_{\mathbb{R}} y^{2} \nu(d y)\right)^{\frac{1}{2}},
$$

and for $i \geq 2$

$$
u^{(i)}(t, x)=\int_{\mathbb{R}} u^{1}(t, x, y) p_{i}(y) \nu(d y) .
$$

Suppose that $u$ is $\mathcal{C}^{1,2}$ function such that $\frac{\partial u}{\partial x}$ and $\frac{\partial^{2} u}{\partial x^{2}}$ is bounded by polynomial function of $x$. Then we have the following

Theorem 5.2: The unique adapted solution of (5.1) is given by

$$
\begin{aligned}
Y_{t} & =u\left(t, X_{t}\right) \\
U_{t} & =\sigma\left(X_{t}\right) \frac{\partial u}{\partial x}\left(t, X_{t}\right) \\
Z_{t}^{(i)} & =\int_{\mathbb{R}} u^{1}\left(t, X_{t-}, y\right) p_{i}(y) \nu(d y) \quad \text { for } i \geq 2, \\
Z_{t}^{(1)} & =\int_{\mathbb{R}} u^{1}\left(t, X_{t-}, y\right) p_{1}(y) \nu(d y)+\frac{\partial u}{\partial x}\left(t, X_{t-}\right)\left(\int_{\mathbb{R}} y^{2} \nu(d y)\right)^{\frac{1}{2}} .
\end{aligned}
$$

Proof: Applying Itô's formula to $u\left(s, X_{s}\right)$ we have

$$
\begin{aligned}
u\left(T, X_{T}\right)-u\left(t, X_{t}\right)= & \int_{t}^{T} \frac{\partial u}{\partial s}\left(s, X_{s}\right) d s+\frac{1}{2} \int_{t}^{T} \sigma^{2}\left(X_{s}\right) \frac{\partial^{2} u}{\partial x^{2}}\left(s, X_{s}\right) d s \\
& +\int_{t}^{T} \sigma\left(X_{s}\right) \frac{\partial u}{\partial x}\left(s, X_{s}\right) d W_{s}+\int_{t}^{T} \frac{\partial u}{\partial x}\left(s, X_{s-}\right) d L_{s} \\
& +\sum_{t<s \leq T}\left[u\left(s, X_{s}\right)-u\left(s, X_{s-}\right)-\frac{\partial u}{\partial x}\left(s, X_{s-}\right) \Delta X_{s}\right] .
\end{aligned}
$$

Lemma 5.1 applied to $u\left(s, X_{s-}+y\right)-u\left(s, X_{s-}\right)-\frac{\partial u}{\partial x}\left(s, X_{s-}\right) y$ gives

$$
\begin{aligned}
& \sum_{t<s \leq T}\left[u\left(s, X_{s}\right)-u\left(s, X_{s-}\right)-\frac{\partial u}{\partial x}\left(s, X_{s-}\right) \Delta X_{s}\right] \\
= & \sum_{i=1}^{\infty} \int_{t}^{T}\left(\int_{\mathbb{R}} u^{1}\left(s, X_{s-}, y\right) p_{i}(y) \nu(d y)\right) d H_{s}^{(i)} \\
& +\int_{t}^{T} \int_{\mathbb{R}} u^{1}\left(s, X_{s-}, y\right) \nu(d y) d s .
\end{aligned}
$$

Exploiting this last identity we obtain

$$
g\left(X_{T}\right)-u\left(t, X_{t}\right)=\frac{1}{2} \int_{t}^{T} \sigma^{2}\left(X_{s}\right) \frac{\partial^{2} u}{\partial x^{2}}\left(s, X_{s}\right) d s
$$




$$
\begin{aligned}
& -\int_{t}^{T} f\left(s, u\left(s, X_{s}\right),\left(u^{(i)}\left(s, X_{s}\right)\right)_{i=1}^{\infty}\right) d s \\
& +\sum_{i=2}^{\infty} \int_{t}^{T}\left(\int_{\mathbb{R}} u^{1}\left(s, X_{s-}, y\right) p_{i}(y) \nu(d y)\right) d H_{s}^{(i)} \\
& +\int_{t}^{T}\left(\int_{\mathbb{R}} u^{1}\left(s, X_{s-}, y\right) p_{1}(y) \nu(d y)\right. \\
& \left.+\left(\frac{\partial u}{\partial x}\left(s, X_{s-}\right)\right)\left(\int_{\mathbb{R}} y^{2} \nu(d y)\right)^{\frac{1}{2}}\right) d H_{s}^{(1)},
\end{aligned}
$$

from which we get the desired result.

\section{Acknowledgments}

We are grateful to the referee for suggestions which allowed us to improve the first version of the paper. We also wish to thank M. Hassani and Y. Ouknine for various discussions on the BSDEs.

\section{References}

[1] K. Bahlali, Backward stochastic differential equations with locally Lipschitz coefficient. C. R. Acad. Sci. Paris Série I, Math. 33 (5), 481-486, (2001).

[2] K. Bahlali, Existence and uniqueness of solutions for backward stochastic differential equations with locally Lipschitz coefficient. Elect. Comm. Proba. 7, 169-180, (2002).

[3] S. Hamadène and J.P. Lepeltier, Zero-sum stochastic differential games and BSDEs. Syst. Cont. Lett. 4, 259-263, (1995).

[4] N. El Karoui, S. Peng and M.C. Quenez, Backward stochastic differential equations in finance. Math. Fina. 1-71, (1997).

[5] A. Løkka, Martingale representation, chaos expansion and Clark-Ocone formulas. MPS-RR 1999-22. MaPhySto (Preprint). (1999).

[6] D. Nualart and W. Schoutens, Chaotic and predictable representations for Lévy processes. Stoc. Proc. Appl. 90 (1) 109-122, (2000).

[7] D. Nualart and W. Schoutens, Backward stochastic differential equations and Feynman-Kac formula for Lévy processes, with applications in finance. Bernoulli (5), 761-776. (2001).

[8] E. Pardoux and S. Peng, Adapted solution of a backward stochastic differential equation. Syst. Cont. Lett. 4, 55-61, (1990).

[9] E. Pardoux, S. Peng, (1992), Backward SDEs and quasilinear PDEs, in: Stochastic Partial Differential Equations and their Applications, (B.L. Rozovskii and R. Sowers, eds.), Lecture Notes and inform. Sci. 176, 200-217. 
[10] S. Peng, (1991), Probabilistic interpretation for systems of quasilinear parabolic partial differential equations, Stoch. Stoch. Reports., 37, 61-74.

[11] P. Protter, Stochastic Integration and Differential Equations. Springer-Verlag. Berlin, (1990).

[12] Y. Ouknine, Reflected BSDE with jumps. Stoc. Stoc. Reports. 5 (1/2), 111-125, (1998).

[13] R. Situ, On solutions of backward stochastic differential equations with jumps and applications. Stoc. Proc. Appl. 6 (2), 209-236, (1997). 


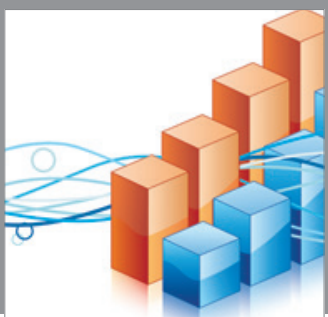

Advances in

Operations Research

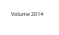

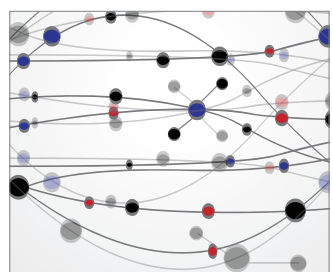

\section{The Scientific} World Journal
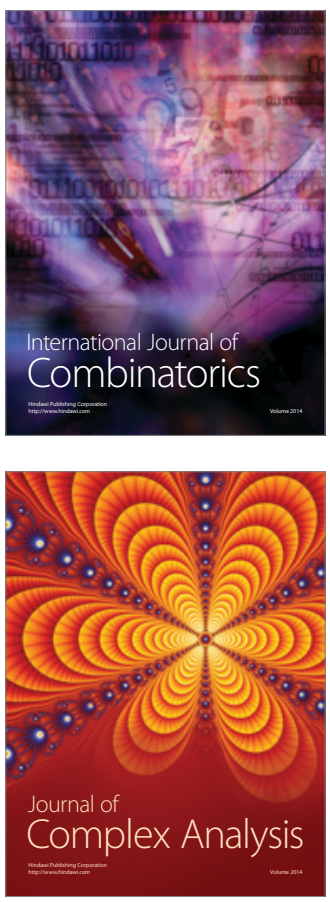

International Journal of

Mathematics and

Mathematical

Sciences
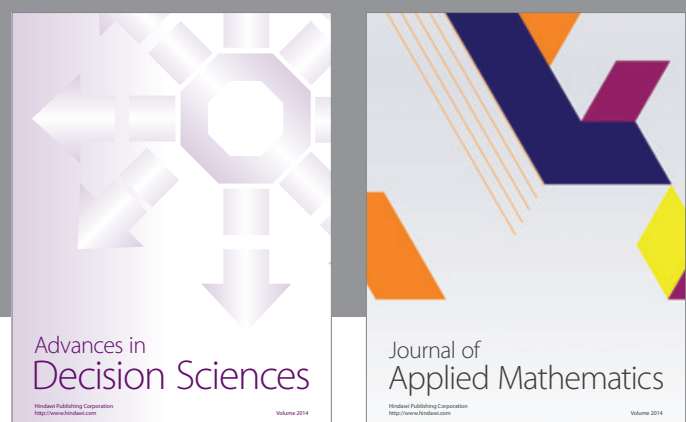

Journal of

Applied Mathematics
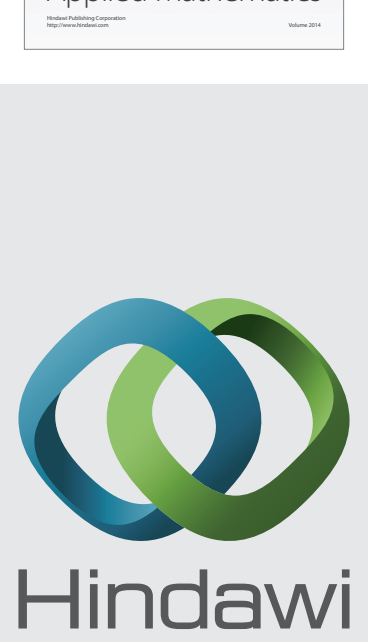

Submit your manuscripts at http://www.hindawi.com
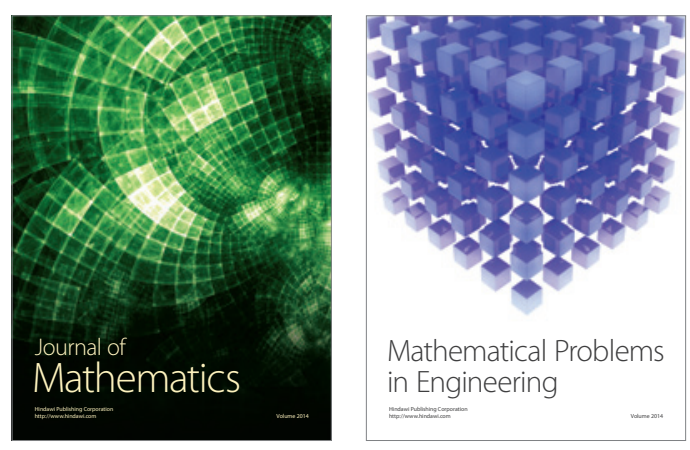

Mathematical Problems in Engineering
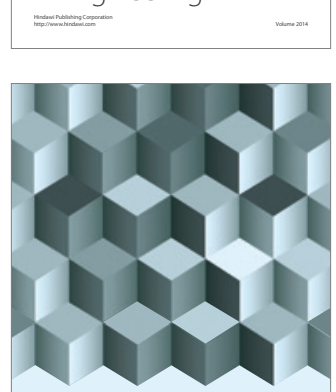

Journal of

Function Spaces
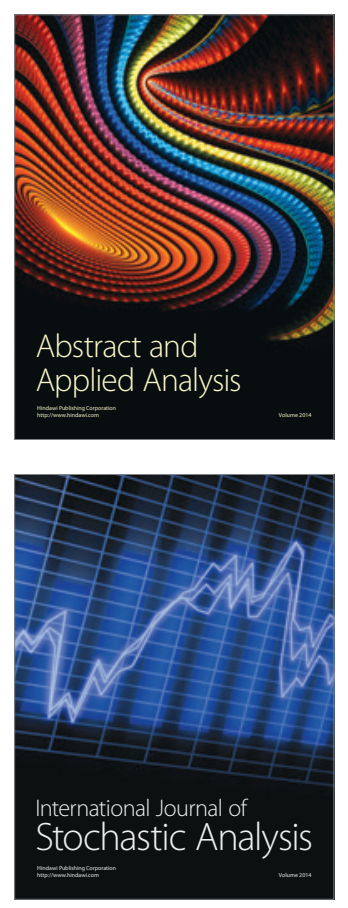

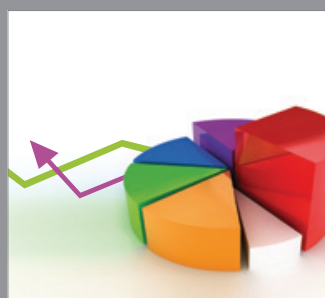

ournal of

Probability and Statistics

Promensencen
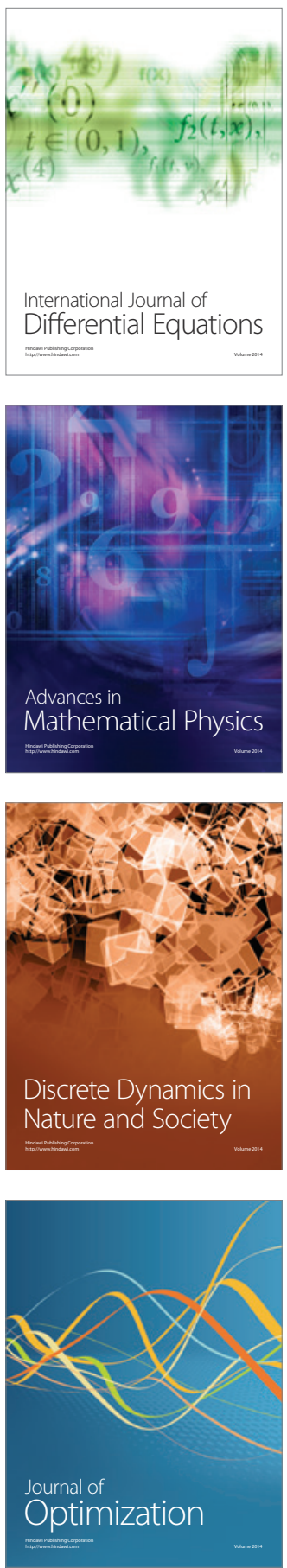